\title{
A safer option to hydroxychloroquine in the chemoprophylaxis of COVID-19 in high- risk health-care workers: A randomized controlled, non-inferiority trial
}

Arvind Chopra ${ }^{1}$, Prasanna Kumar Rao $^{2}$, Govind Reddy ${ }^{3}$, Manohar Gundeti ${ }^{4}$, Kuldeep Choudhary $^{4}$, Muffazal Lakdawala ${ }^{5}$, Manjit Saluja ${ }^{1}$, Sanjay Tamoli ${ }^{6}$, Girish Tillu ${ }^{7}$, Sanjeev Sarmukkaddam ${ }^{1}$, Dilip Gode ${ }^{8}$, Ashwinikumar Raut ${ }^{9}$, BCS Rao ${ }^{10}$, Shruti Khanduri ${ }^{10}$, Narayanam Srikanth ${ }^{10}$, Bhushan Patwardhan ${ }^{7}$

1. Centre for Rheumatic Diseases, Pune, India, 411001

2. Sri Dharmasthala Manjunatheshwara College of Ayurveda \& Hospital, Hassan, India, 573201

3. Regional Ayurveda Research Institute, Nagpur, India, 440009

4. RRAP Central Ayurveda Research Institute, Mumbai, India, 400018

5. H.N. Reliance Foundation Hospital and Research Centre, Mumbai, India, 400004

6. Target Institute of Medical Education and Research, Mumbai, India, 400068

7. Savitribai Phule Pune University, Pune, India, 411007

8. Datta Meghe Institute of Medical Sciences, Nagpur, India, 440016

9. Kasturba Health Society's Medical Research Centre, Mumbai, India, 400056

10. Central Council for Research in Ayurvedic Sciences, New Delhi, India, 110058

\section{Corresponding Author:}

Professor Bhushan Patwardhan, PhD, FNASc, FAMS

National Research Professor-Ayush

Interdisciplinary School of Health Sciences

Savitribai Phule Pune University

Ganeshkhind, Pune 411007, India

Phone: +912025691758

Email: bpatwardhan@gmail.com

Key to Authors:

AC: Arvind Chopra

PKR: Prasanna Kumar Rao

GR: Govind Reddy

MG: Manohar Gundeti

KC: Kuldeep Choudhary

ML: Muffazal Lakdawala

MS: Manjit Saluja

GT: Girish Tillu

SS: Sanjiv Sarmukkaddam

DG: Dilip Gode

AR: Ashwinikumar Raut

BCSR: BCS Rao

SK: Shruti Khanduri

ST: Sanjay Tamoli

NS: Narayanam Srikanth

BP: Bhushan Patwardhan

Word count: Abstract 377 and Main manuscript: 4365 


\begin{abstract}
Objectives: Comparative study of Ashwagandha (Withania somnifera; WS) and hydroxychloroquine (HCQ) for chemoprophylaxis against COVID-19 in actively engaged high-risk health-care workers.

Design: Randomized, multicentric, open label, active control, two arm parallel efficacy study of 16 weeks. Sample size was based on pre-set $15 \%$ non-inferiority margin to HCQ for prophylaxis against COVID-19 with $80 \%$ power and alpha $<0.05$.
\end{abstract}

Participants: 400 health-care workers from three sites who were asymptomatic and tested negative for a quantitative Reverse Transcription Polymerase Chain Reaction test (RT-PCR) for COVID-19 and SARS-CoV-2 antibodies (IgG) were randomized in a 1:1 ratio. Participants observed physical protection measures as per the national policy. All incident confirmed COVID-19 were withdrawn.

Interventions: Two tablets of $250 \mathrm{mg}$ standardized aqueous extract of WS, twice daily after meal or HCQ $800 \mathrm{mg}$ loading followed by $400 \mathrm{mg}$ weekly for 16 weeks as per the national guidelines.

Main outcome measures: The primary efficacy measure was "failure of prophylaxis" as confirmed by RT-PCR at any time during the study. Both intention-to-treat (ITT) and perprotocol (PP) efficacy analyses were performed.

Results: 95 participants in the Ashwagandha (WS) arm and 101 participants in the HCQ arm completed the study. Both groups were well matched at the baseline. 91 participants from the Ashwagandha arm and 84 from the HCQ arm were withdrawn because they received the COVID-19 vaccination. Four participants (2\%; 95\% CI 2.8 to 3.9\%) in the Ashwagandha and 5 (2.5\%; $95 \%$ CI 5.4 to $8 \%$ ) in the HCQ arm developed confirmed COVID-19. This was within the prefixed non-inferiority margin and the $95 \% \mathrm{CI}$ of the absolute risk reduction (ARR) was 2.9 to $3.8 \%$ intention to treat (ITT) and -5.9 to $7.5 \%$ per protocol (PP). The $95 \%$ CI of ARR for the total COVID-19 cases was -2.8 to $11.9 \%$ ITT and -5.7 to $20.3 \%$ PP. Several health measures, particularly anxiety and stress, improved significantly in the Ashwagandha arm. Seven out of 117 in the Ashwagandha and 59 out of 178 in the HCQ groups were reported to be possible drug-related adverse events (AE); there were significantly less gut-related $\mathrm{AE}$ in 
the Ashwagandha group. AE were mostly mild and did not cause withdrawal. All incident COVID-19 cases recovered without complications.

Conclusions: Ashwagandha was non-inferior to HCQ in the chemoprophylaxis against COVID-19 in high risk health-care workers. It was significantly safer, well tolerated and improved quality of life measures. Ashwagandha as COVID-19 prophylaxis seems appropriate in high-risk populations.

Trial registration: The Clinical Trials Registry India Number CTRI/2020/08/027163 dated August 14, 2020.

Keywords: COVID-19, Prophylaxis, Withania somnifera, Ashwagandha, Hydroxychloroquine, Ayurveda, Herbal drugs.

\section{What is already known}

- No specific therapies for COVID-19 are available.

- In addition to vaccines, safer chemoprophylaxis agents are needed.

- Use of HCQ based on empirical data is controversial.

\section{What this study adds}

- Ashwagandha, a known immunomodulator is non-inferior to HCQ.

- Ashwagandha can be used for effective chemoprophylaxis of COVID-19 with safety and health benefits. 


\section{Introduction}

The novel corona virus disease 19 (COVID-19) pandemic requires several immediate desperate measures to control the spread and devastation. There are, currently, no specific therapies and several drugs have been repurposed for prophylaxis and treatment. At the outset, Hydroxychloroquine (HCQ) was posed as an effective solution and was recommended in several countries including India based on empirical evidence. In India, a large number of health-care workers) consumed HCQ as a standard chemoprophylactic measure in accordance with an advisory issued by the Indian Council of Medical Research (ICMR), Government of India. ${ }^{1}$ However, several global reports raised serious concerns about drug toxicity and the inappropriate use of HCQ. Effective and safer drugs were urgently needed especially when vaccines were not available.

India has a legal recognition for traditional, complementary, and integrative medicine (T\&CM) including Ayurveda, Yoga, Unani, Siddha, Homoeopathy (Ayush). The precarious pandemic situation and limitations in modern therapeutics led to a surge of research activities including several drug trials in the domain of $\mathrm{T} \& \mathrm{CM} .^{2-5}$ Millions of Indians were reported to have used T\&CM herbal drugs as home preventive measures. ${ }^{6}$

The Ministry of Ayush, Government of India has recommended the use of Withania somnifera (WS) also known as Ashwagandha, for COVID-19 prophylaxis. ${ }^{7}$ Since ancient historic times, Ayurveda physicians have extensively used Ashwagandha to improve immunity. ${ }^{8}$ Ashwagandha has an immunomodulatory activity and is reported to reduce inflammation and mental stress. ${ }^{9}$ It is shown to be a selective-Th-1 upregulating activity. ${ }^{10}$ Several studies have endorsed long-term safety. ${ }^{11-13}$ Available data indicates that Ashwagandha is a good candidate for clinical repurposing and prophylaxis against COVID-19. ${ }^{14}$

It was against this perspective that we decided to challenge the use of HCQ and suggested Ashwagandha as an effective and safer alternative chemoprophylactic agent for COVID-19. This study was undertaken when vaccines were not available. Agreeably, in the current situation, vaccination remains the most effective prophylaxis. ${ }^{15}$

In this study, Ashwagandha was compared with HCQ for efficacy and safety in the preexposure prophylaxis against COVID-19 among health-care workers. The study was commenced on Sept 15, 2020 and completed on April 30, 2021. After completion of an eightweek trial by 160 participants, an a-priori planned interim analysis was done to assess the 
efficacy of the study drugs and evaluate adverse events (AEs) with emphasis on causality. The interim analysis showed that Ashwagandha was not inferior to HCQ and comparatively, had less drug-related AE. ${ }^{16}$ The data safety monitoring board (DSMB) advised the sponsor to complete the study as per the protocol after careful scrutiny of the interim results.

\section{Methods}

The study design was a prospective, randomized, open label, parallel efficacy, active control, two-arm multicentric drug trial of a duration of 16 weeks. The sample size and primary efficacy was based on a non-inferiority design comparing Ashwagandha as an investigational drug with HCQ as control in health-care workers actively engaged in COVID-19 patient care. The trial was completed at three study sites in the Indian cities Mumbai, Nagpur, and Hassan. All trial participants were jointly examined by a team of Ayurveda and modern medicine physicians at each study site.

The study was funded by the Central Council for Research in Ayurveda Sciences (CCRAS), Ministry of Ayush (MoA), Government of India. The study was carried out in accordance with the principles of Good Clinical Practice (GCP), Declaration of Helsinki (Brazil update 2013), ICMR and CCRAS, MoA Guidelines (2018). ${ }^{17-19}$ The protocol was approved by the Institutional Ethics Committee at each study site and was registered on August 14, 2020 with the Clinical Trial Registry of India, number CTRI/2020/08/027163 (Provided as a

Supplementary File). ${ }^{20}$ The study was monitored by an independent DSMB and the reporting was done in accordance with the CONSORT guidelines. ${ }^{21}$ A checklist relevant to randomized clinical trials based on the CONSORT statement is submitted as a supplementary file. The overall scheme of the study is shown in Fig 1.

\section{Participants}

400 participants signed an informed consent and were enrolled into the study after confirming eligibility during the screening process. All participants were naïve for HCQ and tested negative for SARS-CoV-2 by the standard RT-PCR assay using nose or throat swab and specific anti-SARS-CoV-2 antibodies IgM and/or IgG by standard serology.

The operative definition of health-care workers, exposure to SARS-CoV-2 and risk assessment was according to the advisory issued by the Ministry of Health and Family Welfare, Government of India. ${ }^{22}$ Health-care workers included doctors, nurses, ward attendants, laboratory staff, medical support, and security staff. All participants were required to adhere to 
the hospital protocol requirements and national guidelines for personal protection against SARS-CoV-2 infection, such as face masks and screens, hand washes, sterile gown, and gloves.

\section{Trial Procedures}

Screening, randomization, and physical examination: Participants were screened as per the protocol. A computer software-generated (WinPepi for Windows, version 11.65) randomization schedule in a 1:1 ratio was used by a statistician at each study site. This allocation sequence was used to enrol participants on a first come, first served basis.

The study protocol contained standard procedures to record, classify, monitor, assign causality and report adverse events (AE). All symptoms irrespective of aetiology were to be recorded. Emphasis was given to any symptom that could be related to COVID-19. Participants downloaded a specially designed mobile phone application called COVID KAVACH developed by CRD Pune for the daily monitoring of AE. ${ }^{23}$ Standard serum assay for specific anti-SARS-CoV-2 antibodies IgG was completed in all participants at screening and on completing the study. A Skiagram of the chest and electrocardiography (ECG) was carried out at screening and ECG on completion of study in HCQ arm.

The assessment of general physical health, psychosocial health, and Quality of Life (QOL) was carried out by using a recently developed and in-house validated Health Related-Behaviour Habit and Fitness Questionnaire (HR-BHF CRD, Pune 2020 version); details are provided in Supplementary File S1. The HR-BHF contained nine questions pertained to general health, anxiety, fatigue, energy level, bowel habits, stress, happiness, sleep, and food and appetite. Each question was answered on a $100 \mathrm{~mm}$ horizontal visual analogue scale (VAS), which was anchored at either end $(0$ and $100 \mathrm{~mm})$ for the worst or best outcomes. We used individual question scores (0-100) and total score (0-900) for analysis in this study.

The participants developing confirmed COVID-19 were withdrawn from the study and referred to a COVID treatment facility. However, the study investigator remained in contact with the treating physician to obtain information about progress of the disease and outcomes. The GoI vaccination programme for COVID-19 began in late January 2021, which overlapped with this drug trial. consequently, several participants were withdrawn after receiving the vaccination; as per protocol, follow up continued.

\section{Interventions}

Investigational Drug: Two tablets of $250 \mathrm{mg}$ standardized aqueous extract of Ashwagandha twice daily (equivalent to $5 \mathrm{gm}$ of Ashwagandha dried root powder per day) to be taken with 
warm water after meals. Participants in the Ashwagandha arm consumed the drug daily for 16 weeks. Details of quality control and standardization of Ashwagandha extract are provided as Supplementary File S2.

Active Control Drug: HCQ was dispensed as $400 \mathrm{mg}$ tablets bearing the same batch number centrally procured from market and supplied by the sponsor to all the trial centres. Following a loading dose of $800 \mathrm{mg}$ (400 mg twice a day on Day One), HCQ was continued with $400 \mathrm{mg}$ once a week for 16 weeks.

Concurrent medication: Any other medication was recorded in the study case record form and was continued at the discretion of the primary care physician.

\section{Data collection}

Paper case report forms (CRFs) were completed and entered onto the database at each study site. Data was collected as per the protocol on a daily basis till a participant was discharged from the hospital, following clinical recovery. Data was recorded in the study CRFs at the point of care and later entered into a central electronic database, under the supervision of the chief clinical coordinator of an appointed contract research organization (CRO). Patients were counselled about post-COVID care. COVID KAVACH helped to track the patients daily after their discharge from the hospital, for feedback on any kind of symptoms or untoward events.

\section{Outcomes}

The primary efficacy measure was the proportion of participants with confirmed COVID-19 at any time during the study period. This was akin to "failure of prophylaxes". All participants with typical symptoms of COVID-19 as per protocol were confirmed if they tested positive by RT-PCR assay for SARS-CoV-2, using a nasal and throat swab.

Secondary efficacy measures included all other incident cases suspected of SARS-CoV-2 infection based on AE profile and or testing positive for specific IgG antibodies to SARS-CoV2. Participants reporting any kind of febrile and or respiratory AE were considered as likely diagnosis of COVID-19 (clinical COVID) for the current data analysis irrespective of the clinical judgement of the study investigator. Secondary efficacy included laboratory measures of routine health assessment and special assay including selected cytokines and anti-oxidant markers. The outcome based on HR-BHF questionnaire was also analysed as secondary efficacy. The AE data of each randomized participant was analysed for safety and tolerability. 


\section{Statistical analysis}

This was a non-inferiority study design with $80 \%$ power and an alpha $<0.05$. There was no prior published control data on the prophylactic role of HCQ in health-care workers at the time of this study. The statistical plan was driven by an expert consensus between the chief clinical coordinator and statistician. It was assumed that 7-10\% participants on HCQ may fail prophylaxis. A 15\% non-inferior margin, one sided, was prefixed for Ashwagandha. 180 participants per arm (Type 1 error excluded) would be required. After considering a dropout rate of about $20 \%$, a final sample size of 400 participants, 200 randomized to each arm, was approved.

The data were analysed for central tendencies and 95\% confidence intervals (95\% CI). Appropriate statistical tests were to be carried out to compare treatment groups as per the nature and normality distribution of data. The primary efficacy analysis of non-inferiority was carried out. Both intent-to-treat (ITT) and per protocol (PP) completer analysis was performed when appropriate. Standard statistical software programs were used (GraphPad InStat Version 3.6 and Confidence Interval Analysis software analysis, BMJ Group, London, 2003).

\section{Patient and public involvement}

No patients were involved in setting the research question, or in developing plans for recruitment, design, implementation, and dissemination of the results of this study. The study was conceived under the COVID-19 pandemic when vaccines were not available.

\section{Results}

Allocation, follow-up, and analysis of trial participants in the study is shown in Fig 2. 400 participants were randomized and 196 participants completed the 16-week trial. 174 participants (33 at week 12 and 120 week 16) were withdrawn after they received the vaccine. This was as per the recommendation of the DSMB and a protocol amendment.

Table 1 shows that both the study arms were well matched for several variables. However, the number of doctors were significantly higher in the Ashwagandha group $(\mathrm{p}=0.02)$. There were no obvious imbalances in the study sites as shown in Supplementary File S3. Total 204 (51\%) participants were withdrawn from the study and the details are shown in Fig 2 and Supplementary File S3. None were withdrawn due to any drug-related adverse event. 


\section{Outcomes}

The primary and secondary efficacy analysis pertaining to COVID-19 outcome is shown in Table 2. Four (2\%) participants in the Ashwagandha arm and five (2.5\%) in the HCQ suffered from confirmed COVID-19 by ITT analysis and the difference (95\% CI -2.8 to 3.9\%) was not significant. 4.2\% Ashwagandha and 5\% HCQS suffered from confirmed COVID-19 by PP analysis and the difference (95\% CI -5.9 to $7.5 \%$ ) was not significant. The difference in the incidence of COVID-19 between the two groups by both ITT and PP analyses fell within the pre-fixed non-inferiority margin of $15 \%$, and thereby fulfilled the primary efficacy criteria. The absolute risk reduction (ARR) and the number needed to treat (NNT) did not significantly differ between the groups (Table 2).

19 participants in the Ashwagandha and 24 participants in the HCQ groups suffered from clinical COVID-19 illness. The results with respect to ARR were not significant. Similarly, the ARR was not significantly different for participants with asymptomatic disease (Table 2). Finally, when the total COVID-19 cases were considered, the absolute risk reduction in favour of Ashwagandha was higher, both by ITT and PP analysis, but not statistically significant; correspondingly, the NNT was substantially reduced (Table 2). The "number needed to harm" (NNH) also was reduced with the total COVID-19 (Table 2).

Table 3 shows some features of interest of the nine participants in the study who were diagnosed with confirmed COVID-19 and demonstrated the primary efficacy outcome. During a pre-planned interim analysis carried out at week 8 , the $95 \%$ confidence interval of the difference in proportion of participants contracting COVID-19 in the two arms was $-3.5 \%$ to 9.3 and in favour of the Ashwagandha arm.

\section{Health assessment}

The HR-BHF question and total score in the study arms is shown in Table 4. There were no significant baseline differences between the two arms. The individual and total scores in the Ashwagandha arm were numerically superior. The total score $(\mathrm{p}=0.02)$ and the score for several questions $(\mathrm{p}<0.05)$ was significantly higher in the Ashwagandha arm at week 16 . At week 8 , the participants in the Ashwagandha arm showed a significantly lesser anxiety and higher energy status (Table 4).

\section{Serology}


Serology assay was carried out to detect specific IgG antibody to SARS-CoV-2. On completion of the study, 23 participants tested positive [10 (10.5\%) in the Ashwagandha arm and 13 (12.9\%) in the HCQ arm]. The 95\% CI of the mean difference was -2.4 to 7.5 (intention to treat) and -5.3 to 13.8 (per protocol); the corresponding values were -3 to 8.1 and -6.6 to 14.5 when the number of participants with confirmed COVID-19 were added. All these intervals remained within the prefixed non-inferiority margin in the study.

\section{Safety}

Table 5 and Supplementary File S4 show the adverse event (AE) profile in the study. The AE reported as per the WHO system approach by the study investigators is shown in Supplementary File S4. Several AE were clubbed together under a single syndrome/symptom complex using clinical judgement and after discussion with investigators (PKR, GR, MG) and these are shown in Table 5. Although they were not serious, 76 (38\%) participants in the Ashwagandha and 92 (46\%) participants in the HCQ group suffered from an AE during the study period ( $\mathrm{p}=0.13$ ); correspondingly, 110 and $164 \mathrm{AE}$ were reported. There were no deaths reported. The AE were predominantly mild and episodic in nature and required reassurance and symptomatic treatment. The causality to a study drug was classified as possible/probable in 11 participants in the Ashwagandha group and 39 in the HCQ group; the difference was statistically significant; only 1 definite causality in the HCQ group (Table 6).

There was a four-fold increase in number of participants in the HCQ group who suffered from gut-related $\mathrm{AE}$ (37 versus 14 in WS, p=0.00) and these were mostly related to symptoms of hyperacidity s. The number of AE in the Ashwagandha group seemed to diminish over time (only 4 at week 16) while the number remained high in the HCQ group (Table 6).

More participants in the HCQ (15 versus 6 in the Ashwagandha) recorded a febrile episode, which was often associated with respiratory symptoms, albeit mild and of short duration (less than five days) (Table 6). Specific RT-PCR assay (or SARS-CoV-2) was limited to participants with typical symptoms and a high index of clinical suspicion.

The febrile and/or respiratory events shown in Table 6 were considered by the study physicians to be non-specific or a probable respiratory virus illness and of mild self-limiting nature, and no laboratory diagnostic work up was carried out. But the latter may not be true in the context of the current COVID-19 pandemic and these events could well be compatible with an illness like COVID-19. Later, eight participants, four each group, with the febrile/respiratory AE were found to be seropositive for specific IgG antibody (COVID-19). The number of participants in 
each of the two study groups who suffered from AE that were compatible with a COVID-19 illness were analysed as secondary efficacy measure and results are shown in Table 2.

None of the participants ever complained of symptoms that could be related to a cardiac disorder. ECGs were performed at baseline and on completion of the study in the HCQ group. None of the ECGs showed any abnormality that could be considered to be a drug-related AE or of any clinical significance.

Supplementary File S4 also shows laboratory abnormalities reported as AE. They were mild and did not seem to be of clinical significance. participants in each of the study groups showed increased erythrocyte sedimentation rate (ESR) and a mild drop (1-1.5 gm/dl) in blood haemoglobin. One participant in the Ashwagandha and three participants in the HCQ groups showed elevated serum liver transaminases (less than 1.5 times the upper limit normal). 14 participants in the Ashwagandha arm and 22 participants in the HCQ arm showed an increase in the blood lipid assay (total and or fraction); increase $<20 \%$ of the upper limit of normal. Participants with laboratory abnormalities were advised to follow up with their primary care physician.

\section{Discussion}

A standardized preparation of Ashwagandha was demonstrated to be non-inferior to HCQ as a chemoprophylactic agent in the pre-prophylaxis against COVID-19 in high-risk health-care workers in a randomized, open label, multicentre drug trial of a duration of 16 weeks. Daily oral administration of Ashwagandha proved to be substantially safer than the weekly regimen of HCQ. Ashwagandha also improved the general physical and mental health. 400 consenting volunteers were enrolled in this two-arm study $(80 \%$ power, alpha $<0.05 \%)$. Four participants (2\%) on Ashwagandha and five participants $(2.5 \%)$ on HCQ prophylaxis contracted confirmed (RT-PCR) symptomatic COVID-19; and the difference both by intention to treat and per protocol analysis was non-significant and within the prefixed non-inferiority margin of $15 \%$ (Table 2). The outcome of non-inferiority for Ashwagandha remained consistent when participants with AE compatible with COVID-19 illness (probable cases) and asymptomatic seropositive (anti-SARS-CoV-2 IgG antibody) were considered; higher proportion of positive cases was recorded in the HCQ group (Table 2). The absolute risk reduction with the use of Ashwagandha as compared to HCQ was non-significant and less than $10 \%$ in each of the subgroups of COVID-19 (Table 2). The number needed to treat for benefit was substantially reduced in the case of total COVID-19; 22 by ITT and 14 by PP analysis (non-significant) 
(Table 2). The power of the study was however reduced as a substantial number of participants were withdrawn due to vaccination in the last four weeks of the study (Supplementary File S2).

A distinct advantage of Ashwagandha in the current study was the superior safety and tolerability profile as compared to HCQ (Table 6). Maximum AE were related to the gastrointestinal system and were significantly less with the Ashwagandha arm, including those with a possible/probable causality (Table 6). There were no other safety concerns in the Ashwagandha arm.

The current study was designed with an element of urgency during the peak period of the first pandemic wave in June 2020. ${ }^{24}$ Prophylaxis studies of infectious diseases are highly sensitive to disease frequency in the target population. The current drug trial was spread from September 2020 to April 2021; the second pandemic wave began in late January 2021. Both the states of Maharashtra and Karnataka, where the study sites were located, were significantly affected. ${ }^{25}$ The incidence of confirmed COVID-19 in the Ashwagandha and HCQ groups in the current study was intriguingly low $(<3 \%)$ and this, to the best of our knowledge, seems unprecedented. About $50 \%$ of the health-care workers at risk in the current study were doctors and nurses (Table 1) and a similar proportion was observed in participants with confirmed COVID-19 (Table 3). However, we tend to believe that both Ashwagandha and HCQ were effective prophylactic agents in the study. We further included AE compatible with COVID-19 illness and asymptomatic cases to determine a more likely burden of COVID-19 in the study population (Table 2). All cases of COVID-19 in the current study were mild. However, confirmed COVID-19 may matter more from a point of view of treatment and prognosis.

\section{Other studies}

This study is the first one comparing HCQ with Ashwagandha as an effective chemoprophylaxis. The choice of HCQ as an active control drug in the current study was indeed a pivotal decision and we are aware of the several controversies that have emerged over time to question its therapeutic effectiveness. ${ }^{26}$ Although a meta-analysis of observational studies is reported, we are not aware of any controlled prospective evaluation of HCQ as a chemoprophylactic agent in COVID-19 in an Indian study. ${ }^{27}$ However, several retrospective studies have reported COVID-19 in health-care workers and further provided support to its continued use in the first pandemic wave. $27 \%$ health-care workers tested RT-PCR positive for SARS-CoV-2 infection in a hospital-based study. ${ }^{28} 11 \%$ of the 3711 health-care workers engaged in hospital-based COVID-19 duties suffered from confirmed COVID-19 illness. ${ }^{29}$ 
$16.3 \%$ health-care workers reported SARS-CoV-2 infection in a case-control study and participants on pre-exposure prophylaxis with HCQ were found to have a lower relative risk (95\% CI 0.61 to 0.90$].{ }^{30} 41.5 \%$ health-care workers who were not on HCQ prophylaxis selfreported confirmed COVID-19 in a recently published multicentric retrospective study with a large sample size; health-care workers on HCQ prophylaxis of more than six-week duration were found to have an adjusted Odds Ratio of 0.28 (95\% CI 0.21, 0.37). ${ }^{31}$ The latter study reported a large spectrum of $\mathrm{AE}$ including 110 cardiac $\mathrm{AE}$ that were possibly mild to moderate and were likely related to HCQ.

It is prudent to address the effectiveness of HCQ from a global perspective as well. The results of randomized, double blind, placebo-controlled studies of HCQ, both pre- and post-exposure prophylaxis, were disappointing and led to substantially decreasing the use of the drug across the world. ${ }^{32-36}$ In our opinion and as gleaned from several publications, the interpretation of the primary outcome in each of these studies was limited by several factors that included difficulties in defining a definite COVID-19 outcome, enrolment problems, failure to achieve target sample size (reduced study power), inadequate RT-PCR assays, and stringent outcomes that were more aligned towards development of the vaccine. ${ }^{37}$ There were additional uncertainties about the HCQ dosage and treatment regimen and undue concern with drug toxicity. In a better designed pre-prophylaxis study, 1483 health-care workers (target sample size 3150) took the weekly dose of $\mathrm{HCQ}$, over 12 weeks, and the incidence rate for confirmed/probable COVID-19 (<45\% with RT-PCR assay) was 5.9\% with once-a-week HCQ, 5.9\% with twice-a-week HCQ, and 7.9\% placebo (non-significant). ${ }^{36}$ Needless to say, the COVID situation has substantially differed across the world due to several regional factors and thus comparisons may, at best, be superficial.

Ashwagandha is popularly used in Ayurveda practice in the Indian subcontinent to promote physical and mental health and treat various medical disorders. ${ }^{9,12,38-40}$ It is a widely used botanical of Ayurveda therapeutics indicated for strengthening immunity and preventing diseases. Recently, a large body of experimental evidence has accrued to support this contention. ${ }^{41-46}$ An anti-viral effect, and more recently, against SARS-CoV-2, was also demonstrated for Ashwagandha. ${ }^{47-49}$ Several drug trials for the therapeutic evaluation of Ashwagandha in COVID-19 were registered. ${ }^{5}$ The current study is the first controlled standard clinical evaluation of Ashwagandha in the pre-prophylaxis of SARS-CoV-2 infection in healthcare workers in India. The outcome of this study is applicable to the community at large. 
The decision to use HCQ in the current study seems correct. We did consider placebo but rejected the idea largely because of ethical issues and the fact that HCQ was already in popular clinical use during the first pandemic wave in India. ${ }^{22}$ An equally important pivotal concern in the current study was setting a non-inferiority margin, which has been explained in the section on "statistical analysis" above. There was no prior placebo-controlled data to guide us and we used an expert consensus to safeguard against a placebo response. Our approach was more inclined towards the recommendation of the Europe, Middle East, Africa (EMEA) group. Admittedly, non-inferiority drug trials are complex. ${ }^{37,50}$

\section{Limitations of this study}

There were several limitations other than those imposed by the COVID-19 pandemic. The health-care workers led a very busy and stressful schedule, and must have found it difficult to adhere to the requirements of the study. It is likely that the latter became somewhat easier by using the mobile app in the current study. Drug compliance was an important concern. The use of HCQ as a prophylactic amongst the health-care workers in India reduced substantially by the end of 2020 and this might have adversely influenced the current study. The community also used several popular home and other traditional remedies to stay healthy and free of COVID-19. ${ }^{6}$ The use of specific RT-PCR assay was restricted to participants with suspected COVID-19 symptoms and may not have been positive in some otherwise typical cases. The sensitivity and specificity of RT-PCR assays and serology for specific COVID-19 antibodies are well known. ${ }^{51}$ We may have missed asymptomatic and atypical cases. However, we did include all participants with febrile/ respiratory symptom AE in the efficacy analysis. We performed specific IgG serology only on completion of the study completion. There was a substantial loss of participants to the COVID-19 vaccination drive and this reduced the power of the study. Due to the pandemic situation, there was minimum physical interaction.

\section{Conclusion}

In this randomized controlled prospective statistically designed study of a 16week duration in health care workers, who were actively engaged in COVID-19 duties, Ashwagandha was demonstrated to be non-inferior to HCQ in the pre-prophylaxis against confirmed cases of COVID-19. However, the safety and tolerability of Ashwagandha was substantially superior and with statistically significant less gut-related adverse events, as compared to HCQ. It further showed impressive improvement in the overall health status of the participants. 
Several population sub-groups with high risk for SARS-CoV-2 infection COVID-19 may need to be protected before they receive the vaccination. There is significant incidence of breakthrough infections following vaccination and in this situation, Ashwagandha may offer additional protection. ${ }^{52} \mathrm{We}$ are in the process of beginning a randomized controlled multicentric trial to study the safety and immunogenicity of Ashwagandha co-administration with COVID-19 vaccination. Study protocol has been already registered and published. ${ }^{53}$

\section{Acknowledgements}

A special thanks to Vaidya Dr Rajesh Kotecha, Secretary, Ministry of Ayush (MoA), Government of India (GoI), for his invaluable guidance and inspiration towards completing the current study project and preparing the study publications. We are grateful to senior Vaidya KS Dhiman, former DG CCRAS, GoI for the speedy completion of the drug trial. We thank several research colleagues in the CCRAS - Dr Ravindra Singh, Dr Babita Yadav, Dr BS Sharma and Dr Azeem Ahmad. We acknowledge with gratitude several colleagues from each trial site - Dr Manish Deshmukh, Dr Swati Munde, Dr Pratap Makhija, Dr Alia Rizvi, Prof Wahid Ali, Dr Neeta Warty, Dr Parth Dave and Mr Akash Saggam. Dr Vinay Pawar played an important role in statistical analysis. We acknowledge with gratitude the excellent support from Mr Dimakh Sahasrabuddhe and his colleagues for the smooth and efficient running and administration of the mobile app, COVID KAVACH, during this study. We thank Dr Lal Hingorani, Pharmanza Herbals Pvt Ltd India for ex-gratis supply of the tables of standardized aqueous extract of Ashwagandha used as the investigational drug in this study. Finally, we thank all the study-site paramedical staff and health-care workers with folded hands for their active participation and wholehearted support.

\section{Author Contributions}

AC and MS prepared the protocol with inputs from GT and AR. AC, SS and ST carried out the interpretation of data. BP (senior author) and GT assisted AC in the analysis and in preparing the manuscript. All the lead study investigators (PKR, GR and MG) confirmed the site data and observations used in this report. BP, GT, NS, BCSR, SK, AR and NS wrote the esctions on the standardization, quality control and traditional medicinal use of Ashwagandha. SS and ST carried out the biostatistical analytical data and its verification in several instances. All the authors have full access to the study data and other observations and tables in the current report 
and vouch for the veracity of the data and the study report. The current manuscript has been approved by all the authors. All the authors have consented to submit this manuscript.

\section{Transparency statement}

$\mathrm{AC}$ (the lead author) affirms that the manuscript is an honest, accurate, and transparent account of the study being reported and that no important aspects of the study have been omitted. There are no discrepancies between the study as originally planned and as documented in the protocol. It is important to note that the COVID-19 vaccination programme was begun in India on January 21, 2021. This was not anticipated when designing protocol and this led to several participants having to withdraw.

\section{Funding Statement}

This study was sponsored by Central Council of Research in Ayurvedic Sciences (CCRAS), Ministry of Ayush, Government of India.

\section{Ethical approvals}

The Institutional Ethics Committees of each of the study sites approved the protocols. Details are as follows RRAP Central Ayurveda Research Institute, Mumbai - 03/2020-2021 dated 17 Aug 2020; Sri Dharmasthala Manjunatheshwara College of Ayurveda \& Hospital, Hassan SDM/IEC/120/2020, dated 11 Aug 2020; Datta Meghe Institute of Medical Sciences, Nagpur - (DU)/IEC/2020/9000, dated 11 Aug 2020.

\section{Competing interests}

None of the authors have any financial conflict of interest regarding this study. The authors BCS Rao, Shruti Khanduri, Narayanam Srikanth work in Central Council for Research in Ayurvedic Sciences (CCRAS), Ministry of Ayush (MoA), Government of India (GOI), New Delhi. Ashwinikumar Raut was a member of DSMB. First author Arvind Chopra is the chief clinical coordinator and corresponding author. Bhushan Patwardhan is Chairman, Interdisciplinary Ayush Research \& Development Task Force, MoA.

\section{Procedural matters}

The sponsor did not participate in the preparation of the study protocol. The sponsor had no role in the collection of data, setting up study e-database, statistical analysis and interpretation of the results and the decision to submit the article for publication. The sponsor (CCRAS) arranged the supply of study drug material. The sponsor provided several plant-related details 
of standardization, quality control, and chemistry manufacturing control (CMC) of Ashwagandha standardized extract. The sponsor appointed a contract research organization (CRO) as per the standard operating procedure (SOP) of the GoI. The CRO was responsible for the general administration and logistics of the study, ensure site training and GCP compliance, collect data and enter into a central electronic database. The CRO performed the data lock prior to data analysis. An independent biostatistician, appointed by the CRO, conducted the first run of statistical analysis. SS, an independent senior biostatistician rechecked the pivotal statistical analysis

Amongst the authors, BCSR, SK and NS were senior Ayurvedic physicians and research executives with CCRAS, and MG, KC and GR were Ayurvedic physician scientists working in institutions administered by CCRAS. PKR works in SDM Ayurveda College managed by a charitable trust. All other authors (AC, GT, MS, AR, ST and BP) are independent professionals belonging to Ayurveda or modern medicine or modern science and have no contractual relationship with CCRAS or its institutions.

\section{Data statement}

The data sets were locked after the completion of the trial. After publication, relevant data sets will be submitted by the chief clinical coordinator to the sponsor CCRAS. Specific request for data sharing may be sent to the lead author at arvindchopra60@ hotmail.com

\section{Dissemination}

The authors will disseminate the study results to the public and the communities through various means including scientific meetings, personal communications, social media and press releases.

\section{References}

1 Revised advisory on the use of Hydroxychloroquine (HCQ) as prophylaxis for SARSCoV-2 infection. https://www.icmr.gov.in/pdf/covid/techdoc/V5_Revised_advisory_on_the_ use_of_HCQ_SARS_CoV2_infection.pdf 2020 (Accessed on 10 Sept 2021).

$2 \quad \mathrm{Ng}$ JY. Global research trends at the intersection of coronavirus disease 2019 (COVID-19) and traditional, integrative, and complementary and alternative medicine: a bibliometric analysis. BMC Complement Med Ther. 2020;20(1):353. doi:10.1186/s12906-020-03151-8.

3 Patwardhan B, Chaturvedi S. India in COVID-19 times: modern and wiser. J Ayurveda 
Integr Med. 2020;11(4):367-368. doi:10.1016/j.jaim.2020.12.006.

4 Xiong X, Wang P, Su K, Cho WC, Xing Y. Chinese herbal medicine for coronavirus disease 2019: a systematic review and meta-analysis. Pharmacol Res. 2020;160:105056. doi:10.1016/j.phrs.2020.105056.

5 Bhapkar V, Sawant T, Bhalerao S. A critical analysis of CTRI registered Ayush studies for COVID- 19. J Ayurveda Integr Med. Published online November 2020. doi:10.1016/j.jaim.2020.10.012.

6 Srikanth N, Rana R, Singhal R, et al. Mobile app-reported use of traditional medicine for the maintenance of health in India during the COVID-19 pandemic: crosssectional questionnaire study. JMIRx med. 2021;2(2):e25703. doi:10.2196/25703.

7 Guidelines for Ayurveda Practitioners for COVID-19 by Ministry of Ayush, Govt. of India. https://www.ayush.gov.in/docs/ayurved-guidlines.pdf 2020 (Accessed on 10 Sept 2021).

8 Gogte VM. Ayurvedic pharmacology and therapeutic uses of medicinal plants (Dravyagunavidnyan-translation). The academic team of Bharatiya Vidya Bhavan's SPARC; 2000.

9 Tillu G, Chaturvedi S, Chopra A, Patwardhan B. Public health approach of Ayurveda and Yoga for COVID-19 prophylaxis. J Altern Complement Med. Published online April 2020:acm.2020.0129. doi:10.1089/acm.2020.0129.

10 Bani S, Gautam M, Sheikh FA, et al. Selective Th1 up-regulating activity of Withania somnifera aqueous extract in an experimental system using flow cytometry. $J$ Ethnopharmacol. 2006;107(1):107-115. doi:10.1016/j.jep.2006.02.016.

11 Verma N, Gupta SK, Tiwari S, Mishra AK. Safety of Ashwagandha root extract: a randomized, placebo-controlled, study in healthy volunteers. Complement Ther Med. 2021;57:102642. doi:10.1016/j.ctim.2020.102642.

12 Raut AA, Rege NN, Tadvi FM, et al. Exploratory study to evaluate tolerability, safety, and activity of Ashwagandha (Withania somnifera) in healthy volunteers. J Ayurveda Integr Med. 2012;3(3):111-114. doi:10.4103/0975-9476.100168.

13 Patel SB, Rao NJ, Hingorani LL. Safety assessment of Withania somnifera extract standardized for Withaferin A: Acute and sub-acute toxicity study. J Ayurveda Integr Med. 2016;7(1):30-37. doi:10.1016/j.jaim.2015.08.001

14 Saggam A, Limgaokar K, Borse S, et al. Withania somnifera (L.) Dunal: Opportunity for Clinical Repurposing in COVID-19 Management. Front Pharmacol. 2021;12:623795. doi:10.3389/fphar.2021.623795

15 COVID-19 vaccines: further evidence of success. GOV.UK. https://www.gov.uk/government/news/covid-19-vaccines-further-evidence-ofsuccess (Accessed on 10 Sept 2021).

16 Chopra A, Srikanth N, Patwardhan B. Withania somnifera as a safer option to Hydroxychloroquine in the chemoprophylaxis of COVID-19: results of interim analysis. Complement Ther Med. Published online August 2021:102768. doi:10.1016/j.ctim.2021.102768.

17 Declaration of Helsinki.World Medical Association Declaration of Helsinki —Ethical 
principles for medical research involving human subjects adopted. Vol 16.; 2018. doi:10.1111/ddg.13528.

18 Mathur R, Swaminathan S. National ethical guidelines for biomedical and health research involving human participants, 2017: a commentary. Vol 3.; 2018. doi:10.20529/IJME.2018.065.

19 General Guidelines for Clinical Evaluation of Ayurvedic Interventions by CCRAS, Ministry of Ayush, Government of India. https://www.ayush.gov.in/docs/clinical_evaluation.pdf (Accessed on 10 Sept 2021)20.

20 Chopra A. A Study of Ashwagandha in the prophylaxis against COVID-19 and its benefits on general health in high risk health care workers: a randomized controlled comparison with Hydroxychloroquine Sulphate (HCQS). Clinical Trial Registry India. CTRI/2020/05/025332 [Registered on: 23/05/2020]

21 Gagnier JJ, Boon H, Rochon P, Moher D, Barnes J, Bombardier C. Recommendations for reporting randomized controlled trials of herbal interventions: explanation and elaboration. J Clin Epidemiol. 2006;59(11):1134-1149.

doi:10.1016/j.jclinepi.2005.12.020.

22 Advisory for managing health care workers working in COVID and non-COVID areas of the hospital. 2020;2020(September):1-14.

23 Covid Kavach - Apps on Google Play.

https://play.google.com/store/apps/details?id=com.moa.covidrecorder\&hl=en (Accesse d on 10 Sept 2021).

24 Order by Ministry of Health Affairs to prevent the spread of COVID-

19.;. https://www.mha.gov.in/sites/default/files/MHAorder copy_0.pdf. 2020

(Accessed on 10 Sept 2021).

25 Revised Guidelines on Clinical Management for COVID-

19. https://www.mohfw.gov.in/pdf/RevisedNationalClinicalManagementGuidelinefor COVID1931032020.pdf 2020 (March). (Accessed on 10 Sept 2021).

26 Younis NK, Zareef RO, Al Hassan SN, Bitar F, Eid AH, Arabi M.

Hydroxychloroquine in COVID-19 patients: pros and cons. Front Pharmacol. 2020;11(November). doi:10.3389/fphar.2020.597985.

27 Stricker RB, Fesler MC. Hydroxychloroquine pre-exposure prophylaxis for COVID-19 in healthcare workers from India: a meta-analysis. J Infect Public Health.

2021;14(9):1161-1163. doi:10.1016/j.jiph.2021.08.001

28 Banerjee A, Mukherjee K, Bhattacharjee D, Garai D, Chakraborty R. Status of healthcare workers in relation to COVID-19 infection: aretrospective study in a level 4 COVID hospital in eastern India. J Assoc Physicians India. 2020;68(12):55-57.

29 Mahajan NN, Mathe A, Patokar GA, et al. Prevalence and clinical presentation of COVID-19 among healthcare workers at a dedicated hospital in India. $J$ Assoc Physicians India. 2020;68(12):16-21.

30. Dev N, Meena RC, Gupta DK, Gupta N, Sankar J. Risk factors and frequency of COVID-19 among healthcare workers at a tertiary care centre in India: a case-control study. Trans R Soc Trop Med Hyg. 2021;115(5):551-556. doi:10.1093/trstmh/trab047. 
31 Badyal D, Chandy S, Chugh PK, et al. Hydroxychloroquine for SARS CoV2 Prophylaxis in healthcare workers - A multicentric cohort study assessing effectiveness and safety. J Assoc Physicians India. 2021;69.

32 Abella BS, Jolkovsky EL, Biney BT, et al. Efficacy and safety of Hydroxychloroquine vs placebo for pre-exposure SARS-CoV-2 prophylaxis among health care workers: a randomized clinical trial. JAMA Intern Med. 2021;181(2):195-202.

doi:10.1001/jamainternmed.2020.6319.

33 Boulware DR, Pullen MF, Bangdiwala AS, et al. A randomized trial of Hydroxychloroquine as postexposure prophylaxis for Covid-19. N Engl J Med. 2020;383(6):517-525. doi:10.1056/NEJMoa2016638

34 Barnabas RV, Brown ER, Bershteyn A, et al. Hydroxychloroquine as postexposure prophylaxis to prevent severe acute respiratory syndrome coronavirus 2 infection : arandomized trial. Ann Intern Med. 2021;174(3):344-352. doi:10.7326/M20-6519.

35 Barbosa J, Kaitis D, Freedman R, Le K, Lin X. Clinical outcomes of Hydroxychloroquine in hospitalized patients with COVID-19: a quasi-randomized comparative study. N Engl J Med. 2020;1:8882.

36 Rajasingham R, Bangdiwala AS, Nicol MR, et al. Hydroxychloroquine as preexposure prophylaxis for coronavirus disease 2019 (COVID-19) in healthcare workers: a randomized trial. Clin Infect Dis an Off Publ Infect Dis Soc Am. 2021;72(11):e835-e843. doi:10.1093/cid/ciaa1571.

37 Schumi J, Wittes JT. Through the looking glass: understanding non-inferiority. Trials. 2011;12:106. doi:10.1186/1745-6215-12-106.

38 Patwardhan B, Chavan-gautam P, Gautam M, Tillu G, Chopra A. Ayurveda rasayana in prophylaxis of COVID-19. Curr Sci. 2020;19(8):1158-1160. doi:https://www.currentscience.ac.in/Volumes/118/08/1158.pdf.

39 Ahmad S, Zahiruddin S, Parveen B, et al. Indian medicinal plants and formulations and their potential against COVID-19-preclinical and clinical research. Front Pharmacol. 2020;11:578970. doi:10.3389/fphar.2020.578970.

40 Gogte. Ayurvedic pharmacology and therapeutic uses of medicinal plants (Dravyagunavidnyan- translation). The academic team of Bharatiya Vidya Bhavan's SPARC; 2000.

41 Davis L, Kuttan G. Immunomodulatory activity of Withania somnifera. J Ethnopharmacol. 2000;71(1-2):193-200. doi:10.1016/S0378-8741(99)00206-8.

42 Malik F, Singh J, Khajuria A, et al. A standardized root extract of Withania somnifera and its major constituent withanolide-A elicit humoral and cell-mediated immune responses by up regulation of Th1-dominant polarization in BALB/c mice. Life Sci. 2007;80(16):1525-1538. doi:10.1016/j.lfs.2007.01.029.

43 Kour K, Pandey A, Suri KA, Satti NK, Gupta KK, Bani S. Restoration of stressinduced altered $\mathrm{T}$ cell function and corresponding cytokines patterns by Withanolide A. Int Immunopharmacol. 2009;9(10):1137-1144. doi:10.1016/j.intimp.2009.05.011.

44 Khan S, Malik F, Suri KA, Singh J. Molecular insight into the immune up-regulatory properties of the leaf extract of Ashwagandha and identification of Th1 immunostimulatory chemical entity. Vaccine. 2009;27(43):6080-6087. 
doi:10.1016/j.vaccine.2009.07.011.

45 Mikolai J, Erlandsen A, Murison A, et al. In vivo effects of Ashwagandha (Withania somnifera) extract on the activation of lymphocytes. J Altern Complement Med. 2009;15(4):423-430. doi:10.1089/acm.2008.0215.

46 Sikandan A, Shinomiya T, Nagahara Y. Ashwagandha root extract exerts antiinflammatory effects in $\mathrm{HaCaT}$ cells by inhibiting the MAPK/NF- $\kappa \mathrm{B}$ pathways and by regulating cytokines. Int J Mol Med. 2018;42(1):425-434.

doi:10.3892/ijmm.2018.3608.

47 Cai Z, Zhang G, Tang B, Liu Y, Fu X, Zhang X. Promising anti-influenza properties of active constituent of Withania somnifera ayurvedic herb in targeting neuraminidase of H1N1 influenza: computational study. Cell Biochem Biophys. 2015;72(3):727-739. doi:10.1007/s12013-015-0524-9.

48 Balkrishna A, Pokhrel S, Singh J, Varshney A. Withanone from Withania somnifera may inhibit novel coronavirus (COVID-19) entry by disrupting interactions between viral S-Protein receptor binding domain and host ACE2 receptor. Virol J. Published online March 2020. doi:10.21203/RS.3.RS-17806/V1.

49 Kumar V, Dhanjal JK, Bhargava P, et al. Withanone and withaferin-A are predicted to interact with transmembrane protease serine 2 (TMPRSS2) and block entry of SARSCoV-2 into cells. J Biomol Struct Dyn. Published online May 2020:1-27. doi:10.1080/07391102.2020.1775704.

50 Mo Y, Lim C, Watson JA, White NJ, Cooper BS. Non-adherence in non-inferiority trials: pitfalls and recommendations. BMJ. 2020;370:m2215. doi:10.1136/bmj.m2215.

51 Khatami F, Saatchi M, Zadeh SST, et al. A meta-analysis of accuracy and sensitivity of chest CT and RT-PCR in COVID-19 diagnosis. Sci Rep. 2020;10(1):22402. doi:10.1038/s41598-020-80061-2.

52 Tyagi K, Ghosh A, Nair D, et al. Breakthrough COVID-19 infections after vaccinations in healthcare and other workers in a chronic care medical facility in New Delhi, India. Diabetes Metab Syndr. 2021;15(3):1007-1008.

doi:10.1016/j.dsx.2021.05.001.

53 Chopra A, Chavan-Gautam P, Tillu G, et al. Randomized, double blind, placebo controlled, clinical trial to study co-administration of Ashwagandha on safety, immunogenicity, and protection with COVID-19 vaccine: astudy protocol. medRxiv. Published online 2021. doi:10.1101/2021.07.02.21259886. 
Fig 1. Study procedures and events

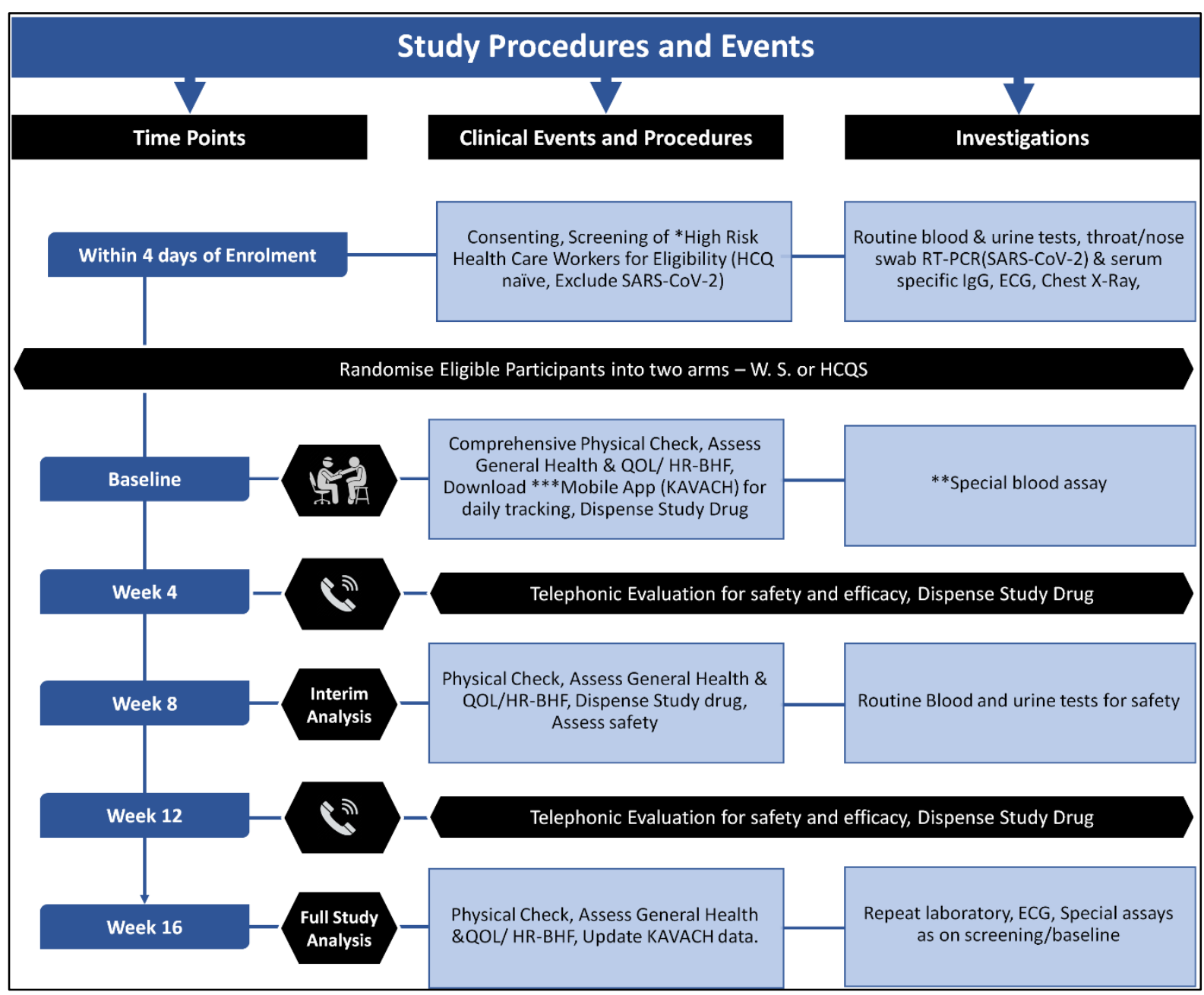


Fig 2: Allocation, follow-up, and analysis of trial participants

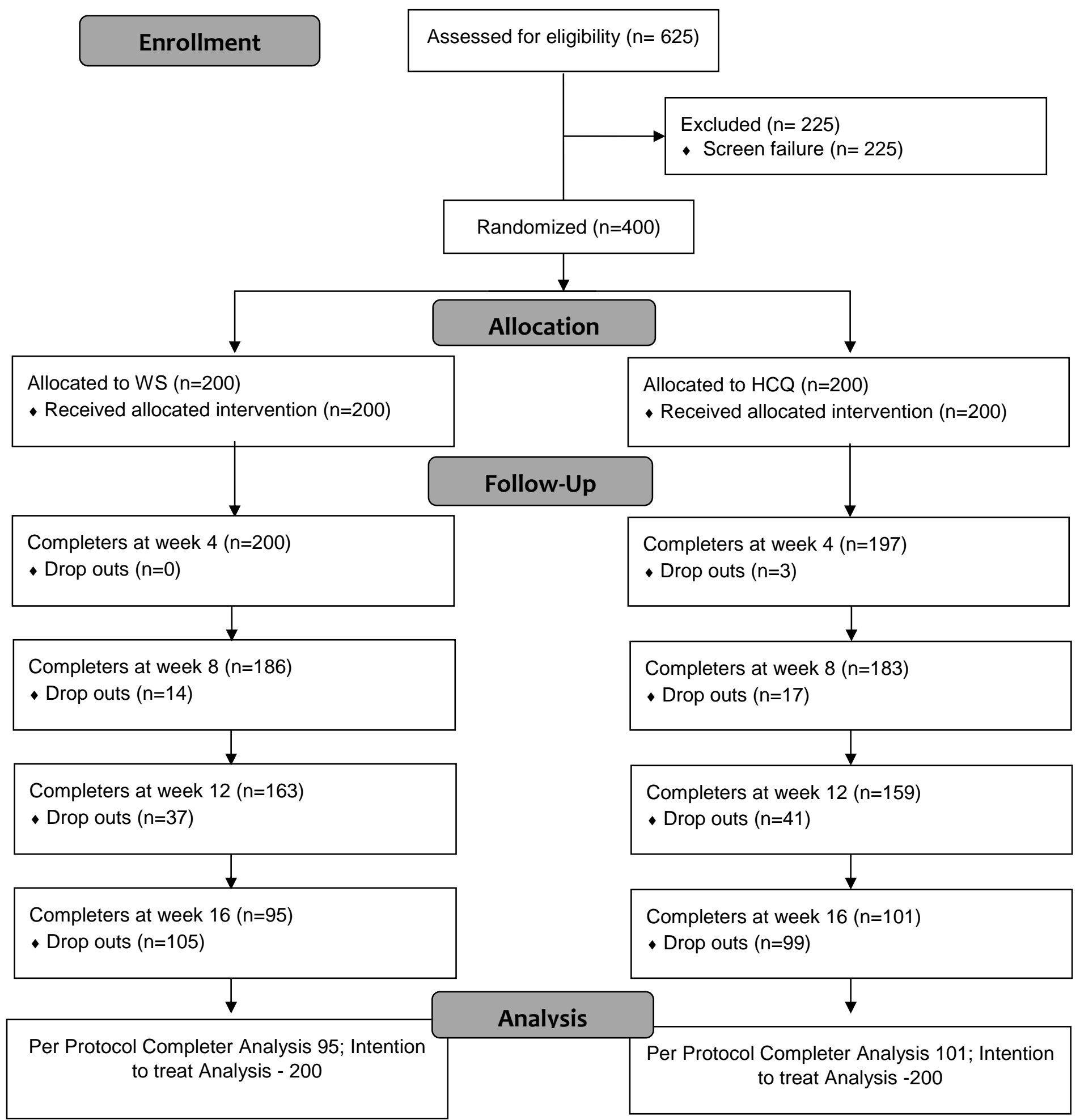


Table 1: Baseline demographic and other features

\begin{tabular}{|c|c|c|c|}
\hline Variable & WS $(n=200)$ & HCQ $(n=200)$ & $\mathrm{p}^{\wedge}$ \\
\hline Age, mean (years, SD) & $35.30 \pm 8.73$ & $34.70 \pm 9.44$ & 0.51 \\
\hline Male, $\mathrm{n}$ (per cent) & $87(43.50 \%)$ & $81(40.50 \%)$ & 0.54 \\
\hline Doctors, $\mathrm{n}$ & $47(23.50 \%)$ & $29(14.5 \%)$ & 0.02 \\
\hline Nurses, $\mathrm{n}$ & $54(27 \%)$ & $67(33.5 \%)$ & 0.15 \\
\hline Ward attendants, $\mathrm{n}$ & $17(8.5 \%)$ & $22(11 \%)$ & 0.40 \\
\hline *Other participants, $\mathrm{n}$ & $82(41 \%)$ & $82(41 \%)$ & 1 \\
\hline Mean BMI $\left(\mathrm{Kg} / \mathrm{M}^{2}, \mathrm{SD}\right)$ & $23.88 \pm 4.41$ & $23.78 \pm 4.41$ & 0.81 \\
\hline Comorbid disorders, $\mathrm{n}$ & $30(15 \%)$ & $21(10.5 \%)$ & 0.17 \\
\hline Diabetes, $\mathrm{n}$ & $9(4.5 \%)$ & $6(3 \%)$ & 0.42 \\
\hline Hypertension, $\mathrm{n}$ & $4(2 \%)$ & $6(3 \%)$ & 0.52 \\
\hline Pre-enrolment exposure, mean (days, SD) & $60.51 \pm 59.16$ & $56.85 \pm 54.49$ & 0.52 \\
\hline Exposure- 8 hours daily (full time job) & $87(43.5 \%)$ & $79(39.5 \%$ & 0.41 \\
\hline$* *$ Exposure- 2-4 hours daily & $15(7.5 \%)$ & 23 & 0.17 \\
\hline **Exposure-2-4 hours, 4-6 times a week & $24(12 \%)$ & $16(8 \%)$ & 0.18 \\
\hline **Exposure-2-4 hours, 2-3 times a week & $74(37 \%)$ & $82(41 \%)$ & 0.41 \\
\hline \multicolumn{4}{|c|}{$\begin{array}{l}\text { Note: WS: Withania somnifera; HCQ: hydroxychloroquine; } \mathrm{n} \text { : Number of participants; SD } \\
\text { standard deviation; }{ }^{\wedge} \text { significant } \mathrm{p}<0.05 \text {, Man Whitney/ } \mathrm{Z} \text { test; } * \text { Other participants: hospita } \\
\text { administration and supervision, food service, security, ambulance drivers; **Exposure } \\
\text { part-time employment }\end{array}$} \\
\hline
\end{tabular}


Table 2: Primary and selected secondary efficacy variables in health-care workers $(\mathrm{n}=400)$ showing number (proportion percentage) and $95 \% \mathrm{CI}$

\begin{tabular}{|c|c|c|c|c|c|}
\hline Variable & WS & HCQS & Total & $\begin{array}{l}\text { Absolute } \\
\text { Risk } \\
\text { Reduction } \\
\text { percent (95\% } \\
\text { CI) }\end{array}$ & $\begin{array}{l}\text { Number Needed to } \\
\text { Treat }(95 \% \mathrm{CI} \text {, } \\
\text { Harm }(\mathrm{H}) \text { to Benefit } \\
\text { (B) }\end{array}$ \\
\hline $\begin{array}{l}\text { Randomized } \\
\text { (ITT) }\end{array}$ & 200 & 200 & 400 & - & - \\
\hline $\begin{array}{l}\text { Study completer } \\
\text { (PP) }\end{array}$ & 95 & 101 & 196 & - & - \\
\hline $\begin{array}{l}* \text { Confirmed } \\
\text { COVID-19 (ITT) }\end{array}$ & $\begin{array}{l}4(2 \%) \\
(0.08,5)\end{array}$ & $\begin{array}{l}5(2.5 \%) \\
(1.1,5.7)\end{array}$ & $\begin{array}{l}9(2.2 \%) \\
(1.2,4.2)\end{array}$ & $0.5(-2.9,3.8)$ & $\begin{array}{l}200(\mathrm{H}=-26 \text { to } \infty \text { to } \\
\mathrm{B}=34)\end{array}$ \\
\hline $\begin{array}{l}\text { *Confirmed } \\
\text { COVID-19 (PP) }\end{array}$ & $\begin{array}{l}4(4.2 \%) \\
(1.6,10.3)\end{array}$ & $\begin{array}{l}5(5 \%) \\
(2.1,11.1)\end{array}$ & $\begin{array}{l}9(4.6 \%) \\
(2.4,8.5)\end{array}$ & $0.7(-5.9,7.5)$ & $\begin{array}{l}135(\mathrm{H}=-13 \text { to } 00 \text { to } \\
\mathrm{B}=27)\end{array}$ \\
\hline $\begin{array}{l}* * \text { AE } \\
\text { compatible with } \\
\text { COVID-19 } \\
\text { illness (ITT) }\end{array}$ & $\begin{array}{l}19(9.5 \%) \\
(6.2,14.4)\end{array}$ & $\begin{array}{l}24(12 \%) \\
(8.2,17.2)\end{array}$ & $\begin{array}{l}43(10.75 \%) \\
(8.1,14.2)\end{array}$ & $2.5(-3.7,8.7)$ & $\begin{array}{l}40(\mathrm{H}=-11 \text { to } 00 \text { to } \\
\mathrm{B}=27)\end{array}$ \\
\hline $\begin{array}{l}* * \mathrm{AE} \\
\text { compatible with } \\
\text { COVID-19 } \\
\text { illness (PP) }\end{array}$ & $\begin{array}{l}19(20 \%) \\
(13.2,29.7)\end{array}$ & $\begin{array}{l}24(23.8 \%) \\
(16.5,32.9)\end{array}$ & $\begin{array}{l}43(21.9 \%) \\
(16.7,28.2)\end{array}$ & $\begin{array}{l}3.8(-7.7 \\
15.4)\end{array}$ & $\begin{array}{l}27(\mathrm{H}=-6 \text { to } 00 \text { to } \\
\mathrm{B}=13)\end{array}$ \\
\hline $\begin{array}{l}\text { Asymptomatic } \\
\text { (Anti-SARS- } \\
\text { CoV-2 IgG + } \\
\text { ITT) }\end{array}$ & $\begin{array}{l}6(3 \%) \\
(1.4,6.4)\end{array}$ & $\begin{array}{l}9(4.5 \%) \\
(2.4,8.3)\end{array}$ & $\begin{array}{l}15(3.7 \%) \\
(2.3,6.1)\end{array}$ & $1.5(-2.7,5.5)$ & $\begin{array}{l}67(\mathrm{H}=-18 \text { to } \propto, \text { to } \\
\mathrm{B}=38)\end{array}$ \\
\hline $\begin{array}{l}\text { Asymptomatic } \\
\text { (Anti-SARS- } \\
\text { CoV-2 IgG +PP) }\end{array}$ & $\begin{array}{l}6(6.3 \%) \\
(2.9,13.1)\end{array}$ & $\begin{array}{l}9(8.9 \%) \\
(4.8,16.1)\end{array}$ & $\begin{array}{l}15(7.7) \\
(4.7,12.2)\end{array}$ & $\begin{array}{l}2.6(-5.3 \\
10.5)\end{array}$ & $\begin{array}{l}38(\mathrm{H}=-9 \text { to } 00, \text { to } \\
\mathrm{B}=19)\end{array}$ \\
\hline $\begin{array}{l}\text { Total COVID-19 } \\
\text { (ITT) }\end{array}$ & $\begin{array}{l}29(14.5) \\
(10.3,20)\end{array}$ & $\begin{array}{l}38(19) \\
(14.2,25)\end{array}$ & $\begin{array}{l}67(16.8 \%) \\
(13.4,20.7)\end{array}$ & $\begin{array}{l}4.5(-2.8, \\
11.9)\end{array}$ & $\begin{array}{l}22(H=-8 \text { to } 00, \text { to } \\
B=35)\end{array}$ \\
\hline $\begin{array}{l}\text { Total COVID-19 } \\
\text { (PP) }\end{array}$ & $\begin{array}{l}29(28.7 \%) \\
(20.8,38.2)\end{array}$ & $\begin{array}{l}38(40 \%) \\
(30.7,50.1)\end{array}$ & $\begin{array}{l}67(34.2 \%) \\
(27.9,41.1)\end{array}$ & $\begin{array}{l}7.1(-5.7, \\
20.3)\end{array}$ & $\begin{array}{l}14(H=-5 \text { to } \infty, \text { to } \\
B=17)\end{array}$ \\
\hline \multicolumn{5}{|c|}{$\begin{array}{l}\text { N:number of participants; WS: Withania somnifera; HCQ: Hydroxychloroquine; } \\
\text { ITT: intention to treat analysis based on randomized sample; PP: per protocol } \\
\text { analysis based on study completers at week 16; CI: confidence interval; } \\
\text { *:Primary efficacy measure, typical clinical phenotype with standard RT-PCR } \\
\text { assay for SARS-CoV-2 positive on nasal/throat swab; ** AE (adverse event) } \\
\text { Symptoms pertain to fever and/or respiratory events compatible with a COVID- } \\
19 \text { illness diagnosis (standard RT-PCR assay not performed, } 6 \text { seropositive for } \\
\text { specific IgG antibody); +: seropositive; See text for details }\end{array}$} & \\
\hline
\end{tabular}


Table 3: Description of participants with confirmed COVID-19 $(n=9)$ and primary efficacy analysis on study completion at week 16

\begin{tabular}{|c|c|c|}
\hline Variable & WS $(n=200)$ & $\operatorname{HCQ}(n=200)$ \\
\hline COVID-19, $\mathrm{n}$ & $4(2 \%)$ & $5(2.5 \%)$ \\
\hline Age range, years & $27-36$ & $31-50$ \\
\hline Men, $\mathrm{n}$ & 2 & 2 \\
\hline Doctor/nurse & 2 & 3 \\
\hline $\begin{array}{l}\text { *Time points of onset after } \\
\text { randomization, weeks }\end{array}$ & $6,9,11,11$ & $3,3,9,9,13$ \\
\hline Period of onset of illness & Oct 2020-Jan 2021 & Oct 2020-Jan 2021 \\
\hline$* *$ Intention to treat analysis & \multicolumn{2}{|c|}{$95 \%$ confidence interval, -2.8 to 3.9} \\
\hline$* *$ Completer analysis & \multicolumn{2}{|c|}{$95 \%$ confidence interval, -5.4 to 8} \\
\hline \multicolumn{3}{|c|}{$\begin{array}{l}\text { Note: } * 2 \text { participants in the Withania somnifera arm with onset at week } 11 \text { were a married } \\
\text { couple and had several members in the close family suffering from COVID-19; none of the } \\
\text { other study participants with COVID-19 had a close family member with COVID-19 during } \\
\text { the study period; n: number of participants; HCQS: Hydroxychloroquine sulfate arm; **; } \\
\text { difference in the proportion of participants with COVID-19 in the two study arms (preset } \\
\text { non-inferiority margin for Withania somnifera compared to HCQ was } 15 \% \text { ); see text for } \\
\text { details }\end{array}$} \\
\hline
\end{tabular}


Table 4: Quality of Life - Health Related Behaviour Habit Fitness

\begin{tabular}{|c|c|c|c|c|c|c|}
\hline \multirow{3}{*}{$\begin{array}{l}\text { Study Group/ } \\
\text { Variable }\end{array}$} & \multicolumn{3}{|l|}{ WS } & \multicolumn{3}{|l|}{ HCQ } \\
\hline & 0 week & $8^{\text {th }}$ week & $16^{\text {th }}$ week & 0 week & $8^{\text {th }}$ week & $16^{\text {th }}$ week \\
\hline & $(n=200)$ & $(n=186)$ & $(n=95)$ & $(n=200)$ & $(\mathrm{n}=183)$ & $(n=101)$ \\
\hline General health & $87.6(11.6)$ & $88.1(12.3)$ & $89.2(10)$ & $87.5(10.6)$ & $89.1(8.6)$ & $87.6(10.6)$ \\
\hline Anxiety & 71.7 (29.4) & $75.9(28.6)^{*}$ & $72.6(31.4)^{*}$ & $72.4(28.1)$ & $68.7(32.1)$ & $64(32)$ \\
\hline Fatigue & $67.4(29.0)$ & $73.3(26.5)$ & $68.4(31.0)$ & $67.9(29.4)$ & $73.2(25.9)$ & $62.7(31.4)$ \\
\hline Energy & $83.6(15.7)$ & $88.0(11.6)^{*}$ & $86.5(16.1)^{*}$ & $83.8(15.5)$ & $85.5(15.4)$ & $78(23.6)$ \\
\hline Bowel habit & 87.7 (13.7) & $89(13.5)$ & $90.2(10.3)^{*}$ & 88.1 (13.0) & $89.1(12)$ & $85.4(18.5)$ \\
\hline Stress & $70.1(28.1)$ & $76.2(25.6)$ & $76.1(24.1)^{*}$ & $72.2(26.4)$ & $75.7(25.1)$ & $68.4(29.3)$ \\
\hline Happiness & $86.1(14)$ & $87.7(14)$ & $88.6(11.1)^{*}$ & 86.7 (12.5) & $87.1(15.3)$ & $84.4(16.5)$ \\
\hline Sleep & $66.8(33.1)$ & $68.3(33.1)$ & $66.3(33.8)$ & $67.3(33.8)$ & $63.4(35.4)$ & $70.2(30.5)$ \\
\hline Appetite & $85(17)$ & $88.7(11.1)$ & 87.7 (12.8) & 85.7 (15.4) & $88.3(12.9)$ & $85.3(15.9)$ \\
\hline Total score & $\begin{array}{l}705.8 \\
(121.3)\end{array}$ & $\begin{array}{l}735.3 \\
(116.6)\end{array}$ & $\begin{array}{l}725.7 \\
*(118.5)\end{array}$ & $\begin{array}{l}711.8 \\
(116.6)\end{array}$ & $\begin{array}{l}720.2 \\
(107.6)\end{array}$ & $\begin{array}{l}686 \\
(124.2)\end{array}$ \\
\hline $\begin{array}{l}\text { Note: } \mathrm{n} \text { : numb } \\
100 \mathrm{~mm} \text { visual } \\
\text { of all } 9 \text { HR-BH } \\
\text { HCQ; see text }\end{array}$ & participa & ? & $\because 1$ & S TID & . & $\begin{array}{l}\text { variable:0- } \\
\text { m of scores } \\
\text { nnifera and }\end{array}$ \\
\hline
\end{tabular}


Table 5: Adverse events (AE)

\begin{tabular}{|c|c|c|c|c|c|}
\hline & \multicolumn{2}{|c|}{ WS $(n=200)$} & \multicolumn{2}{|c|}{ HCQ (n=200) } & \multirow{2}{*}{$\begin{array}{l}\text { *P value } \\
\text { Comparing } \\
\text { participants }\end{array}$} \\
\hline Event Description & $\begin{array}{l}\text { Number of } \\
\text { participants }\end{array}$ & $\begin{array}{c}\text { Number of } \\
\text { AEs }\end{array}$ & $\begin{array}{l}\text { Number of } \\
\text { participants }\end{array}$ & $\begin{array}{c}\text { Number of } \\
\text { AEs }\end{array}$ & \\
\hline Total AE & 76 & 114 & 92 & 162 & 0.13 \\
\hline Total GIT & 14 & 15 & 37 & 53 & 0.00 \\
\hline Total febrile & 6 & 6 & 11 & 11 & 0.32 \\
\hline Total respiratory & 11 & 12 & 9 & 9 & 0.82 \\
\hline Febrile-viral fever & 2 & 2 & 3 & 3 & - \\
\hline Febrile-(NRI-MSK) & 1 & 1 & 2 & 2 & - \\
\hline Fever-nonspecific & 1 & 1 & 0 & 0 & - . \\
\hline Fever-URTI & 0 & 0 & 4 & 4 & - \\
\hline Fever-LRTI & 1 & 1 & 1 & 1 & - \\
\hline Fever-RTI & 1 & 1 & 1 & 1 & - \\
\hline Afebrile URTI & 5 & 6 & 6 & 6 & - \\
\hline Afebrile RTI & 2 & 2 & 0 & 0 & - \\
\hline Cough (Non-specific episodic) & 3 & 3 & 0 & 0 & - \\
\hline $\begin{array}{l}\text { Breathlessness non-specific } \\
\text { episodic }\end{array}$ & 1 & 1 & 3 & 3 & 0.62 \\
\hline Hyperacidity-related symptom & 6 & 6 & 23 & 30 & 0.00 \\
\hline Gastritis & 3 & 3 & 11 & 11 & 0.05 \\
\hline Nausea & 1 & 2 & 2 & 2 & - \\
\hline Dyspepsia & 0 & 0 & 3 & 3 & - \\
\hline Gastroenteritis & 0 & 0 & 2 & 2 & - \\
\hline
\end{tabular}




\begin{tabular}{|c|c|c|c|c|c|}
\hline Anorexia & 2 & 2 & 2 & 2 & - \\
\hline Total AE (GIT) Week 4 & 4 & 4 & 14 & 15 & 0.03 \\
\hline Total AE (GIT) Week 8 & 6 & 6 & 10 & 11 & 0.04 \\
\hline Total AE (GIT) Week 12 & 2 & 2 & 10 & 13 & 0.04 \\
\hline Total AE (GIT) Week 16 & 3 & 3 & 14 & 14 & 0.08 \\
\hline Causality -Not related (GIT) & 7 & 7 & 5 & 5 & 0.77 \\
\hline Causality- Unlikely (GIT) & 5 & 6 & 9 & 11 & 0.42 \\
\hline Causality-Possible (GIT) & 1 & 1 & 19 & 24 & 0.00 \\
\hline Causality-Probable (GIT) & 1 & 1 & 10 & 12 & 0.01 \\
\hline Causality-Definite (GIT) & 0 & 0 & 1 & 1 & - \\
\hline \multicolumn{6}{|c|}{$\begin{array}{l}\text { Note: n: number of participants; WS: Withania somnifera; GIT: gastrointestinal tract AE (participants and } \\
\text { events) shown in parenthesis; } 1 \text { GIT-AE each in HCQ- hypersalivation, mouth ulcer and constipation; } 1 \text { GIT- } \\
\text { AE each in Ashwagandha-mouth ulcer, constipation; URTI: upper respiratory tract infection; LRTI: lower } \\
\text { respiratory tract infection; *p value: Chi-square statistic, Fisher exact test, significant p<0.05; See Text for } \\
\text { detail }\end{array}$} \\
\hline
\end{tabular}

